\title{
Growth, Optical and Electrical Properties of L-Prolinium Tartrate NLO Single Crystals
}

\author{
S.Suresh \\ Department of Physics \\ Loyola College, Chennai-34
}

\author{
P.Mani \\ Department of Physics \\ Hindustan Institute of \\ Technology, Padur
}

\author{
K.Anand \\ Department of Physics \\ Manakula Vinayagar Institute of \\ Technology, Pondicherry
}

\begin{abstract}
Single crystal of L-Prolinium Tartrate (LPT) was grown from aqueous solution by slow evaporation technique. The crystalline nature of the material has been confirmed by single crystal X-ray diffraction. The optical transmission study reveals the transparency of the crystal in the entire visible region and the cut off wavelength has been found to be 220 $\mathrm{nm}$. The optical band gap is found to be $3.60 \mathrm{eV}$. The transmittance of LPT crystal has been used to calculate the refractive index $n$, the extinction coefficient $K$ and both the real $\varepsilon_{\mathrm{r}}$ and imaginary $\varepsilon_{\mathrm{i}}$ components of the dielectric constant as functions of wavelength. The AC and DC conductivity studies of the crystals were carried out to determine the activation energy for conduction process. The photoconductivity studies reveal that the crystal exhibits negative photoconductivity.
\end{abstract}

\section{Keywords}

Single crystal, slow evaporation technique, Single crystal Xray diffraction, $\mathrm{AC}$ and $\mathrm{DC}$ conductivity studies and Photoconductivity studies.

\section{INTRODUCTION}

Recently, the development of potential nonlinear optical (NLO) crystals, capable of generating green and blue laser light is of great importance for frequency conversion, optical data storage, optical amplifiers, image processing, integrated optics, etc. [1]. Nonlinear optics has been an active field of research with the advent of lasers followed by the demonstration of harmonic generation in Quartz [2]. Photonic crystals, which can guide light in the similar way as semiconductors manipulate electrons, can be used to carry, store and process informations [3]. Materials in single crystalline forms are required for the construction of photonic and NLO devices with high performance. Semiorganic crystals, which have the combined properties of inorganics and organics, are of current interest due to their high thermal and mechanical stabilities in addition to nonlinearity. The present Investigation, analyses the growth, optical, electrical and photoconductivity properties of LPT single crystal.

\section{CRYSTAL GROWTH}

Single crystals of LPT were grown from L-Proline and LTartaric acid taken in equimolar ratio in aqueous solution by slow evaporation method. The solution was stirred continuously using a magnetic stirrer. The prepared solution was filtered and kept undisturbed at room temperature. Tiny seed crystals with good transparency were obtained due to the spontaneous nucleation. Among them, defect free seed crystal was suspended in the mother solution, which was allowed to evaporate at room temperature. Large size single crystals were obtained due to collection of monomers at the seed crystal sites from the mother solution after the nucleation process was completed. Figure 1 shows as-grown crystals of LPT.

\section{RESULTS AND DISCUSSION}

\subsection{Single crystal $X$-ray diffraction}

Single crystal X-ray diffraction analysis for the grown crystals has been carried out to identify the cell parameters using an ENRAF NONIUS CAD 4 automatic X-ray diffractometer. Calculated lattice parameters are: $\mathrm{a}=5.0013 \AA$, $\mathrm{b}=17.6874$ $\AA, \mathrm{c}=6.5268 \AA, \beta=100.45^{\circ}$ and the space group is $\mathrm{P} 2{ }_{1}$. These values are found to agree with the reported values [4].

\subsection{Optical Study}

The UV-Vis-NIR spectrum of LPT was recorded with a Varian Cary 5E spectrophotometer in the range 200-1200 nm with a crystal of thickness of $2 \mathrm{~mm}$. From the spectrum (Figure 2), it is evident that LPT crystal has UV cut off wavelength at $220 \mathrm{~nm}$, which is sufficient for SHG laser radiation of $1064 \mathrm{~nm}$ or other applications in the blue region. It further indicates that the crystal has wide transparency window between $200 \mathrm{~nm}$ and $1200 \mathrm{~nm}$. The wide transparency in the entire visible region is one of the additional key requirements for having efficient NLO character. The measured transmittance $(T)$ was used to calculate the absorption coefficient $(\alpha)$ using the formula

$$
\alpha=\frac{2.3026 \log \left(\frac{1}{T}\right)}{t}
$$

where $t$ is the thickness of the sample. Optical band gap $\left(\mathrm{E}_{\mathrm{g}}\right)$ was evaluated from the transmission spectrum and optical absorption coefficient $(\alpha)$ near the absorption edge is using the formula [5]

$$
\alpha h v=A\left(h v-E_{g}\right)^{1 / 2}
$$

where $A$ is a constant, $E_{g}$ the optical band gap, h the Planck constant and $\mathrm{n}$ the frequency of the incident photons. The band gap of LPT crystal was estimated by plotting $(\alpha h v)^{1 / 2}$ versus $h v$ as shown in Figure 3 and extrapolating the linear portion near the onset of absorption 
edge to the energy axis. From the figure, the value of band gap was found to be $3.60 \mathrm{eV}$. Extinction coefficient $(K)$ can be obtained from the equation,

$$
K=\frac{\lambda \alpha}{4 \pi}
$$

The transmittance $(T)$ is given by

$$
T=\frac{(1-R)^{2} \exp (-\alpha t)}{1-R^{2} \exp (-2 \alpha t)}
$$

Reflectance $(R)$ in terms of absorption coefficient can be obtained from the above equation. Hence,

$$
R=\frac{\exp (-\alpha t) \pm \sqrt{\exp (-\alpha t) T-\exp (-3 \alpha t) T+\exp (-2 \alpha t) T^{2}}}{\exp (-\alpha t)+\exp (-2 \alpha t) T}
$$

Refractive index $(n)$ can be determined from reflectance data using the equation,

$$
n=-(R+1) \pm 2 \frac{\sqrt{R}}{(R-1)}
$$

The refractive index (n) is 1.42 at $\lambda=1100 \mathrm{~nm}$.

From the optical constants, electric susceptibility $\left(\chi_{c}\right)$ can be calculated using the following relation [6]

$$
\varepsilon_{r}=\varepsilon_{0}+4 \pi \chi_{C}=n^{2}-k^{2}
$$

Hence,

$$
\chi_{C}=\frac{n^{2}-k^{2}-\varepsilon_{0}}{4 \pi}
$$

where $\varepsilon_{0}$ is the dielectric constant in the absence of any contribution from free carriers. The value of electric susceptibility $\chi_{C}$ is 0.143 at $\lambda=1100 \mathrm{~nm}$. The real part of the dielectric constant $\varepsilon_{r}$ and the imaginary part of the dielectric constant $\varepsilon_{i}$ can be calculated from the relations [7],

$$
\varepsilon_{r}=n^{2}-k^{2} \quad \& \quad \varepsilon_{i}=2 n k
$$

The value of $\varepsilon_{r}$ and $\varepsilon_{i}$ at $\lambda=1100 \mathrm{~nm}$ are 1.42 and $6.324 \times 10^{-5}$ respectively. The optical study is useful to get the information regarding the transparent region, the optical band gap and the dielectric behavior of the grown crystals.

\subsection{Electrical Conductivity Measurements}

\subsubsection{AC Conductivity Study}

The ac conductivity study of the LPT crystal was carried out at $1 \mathrm{KHz}$. In the high temperature (Intrinsic) region, the effect of impurity on electrical conduction has not made any appreciable change whereas in the low temperature (extrinsic) region, the presence of impurity in the crystal has an impact and particularly increases its conductivity. The electrical conduction in dielectrics is mainly a defect controlled process in the low temperature region (Figure 4).The presence of impurities and vacancies predominantly determine this region. The energy needed to form the defect is much larger than the energy need for its drift. It is found from the Arrhenius plot (Figure 5) for the LPT crystal that the conductivity increases with temperature and the value of activation energy is found to be $0.120 \mathrm{eV}$.

\section{3.3.2 DC Conductivity Study}

The experiment was carried out using the conventional twoprobe technique at different temperature ranging from 313 to $423 \mathrm{~K}$, and sufficiently sized crystal of LPT was used for the conductivity study. The crystal was perfectly cut into rectangular slaps and then polished using silicon carbide paper. Figure 6 shows the dependence of dc conductivity of the grown crystal LPT on the temperature. The activation energy of the crystal for the dc conductivity is found to be $0.050 \mathrm{eV}$ (Figure 7). The variation of dielectric properties with respect to frequency and temperature reveals the normal dielectric behavior of the material. It is evident from electrical conductivity of the material that the rotation of ions is responsible for conduction process. Similarly when a powerful electromagnetic radiation (laser beam) is incident on the medium, the rotation of ions gives rise to induced polarization for changing the wavelength or frequency of the incident radiation leading to remarkable behavior (NLO) of the grown material.

\subsection{Photoconductivity Property}

Photoconductivity measurements were made using Keithley 485 picoammeter. The dark current was recorded by keeping the sample unexposed to any radiation. Figure 8 shows the variation of both dark current $\left(\mathrm{I}_{\mathrm{d}}\right)$ and photocurrent $\left(\mathrm{I}_{\mathrm{p}}\right)$ with applied field. It is seen from the plots that both $I_{d}$ and $I_{p}$ of the sample increase linearly with applied field. It is observed from the plot that the dark current is always higher than the photo current, thus confirming the negative photoconductivity nature of the material. 


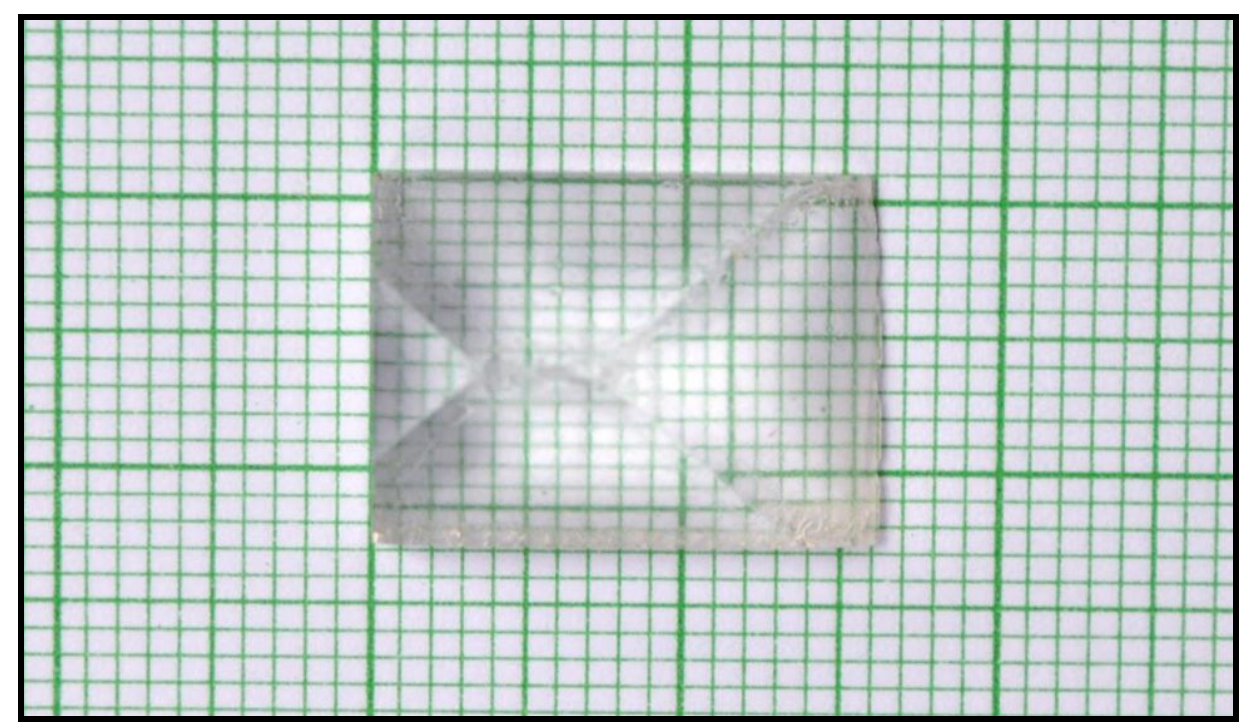

Figure .1 As grown L-Prolinium tartrate single crystal

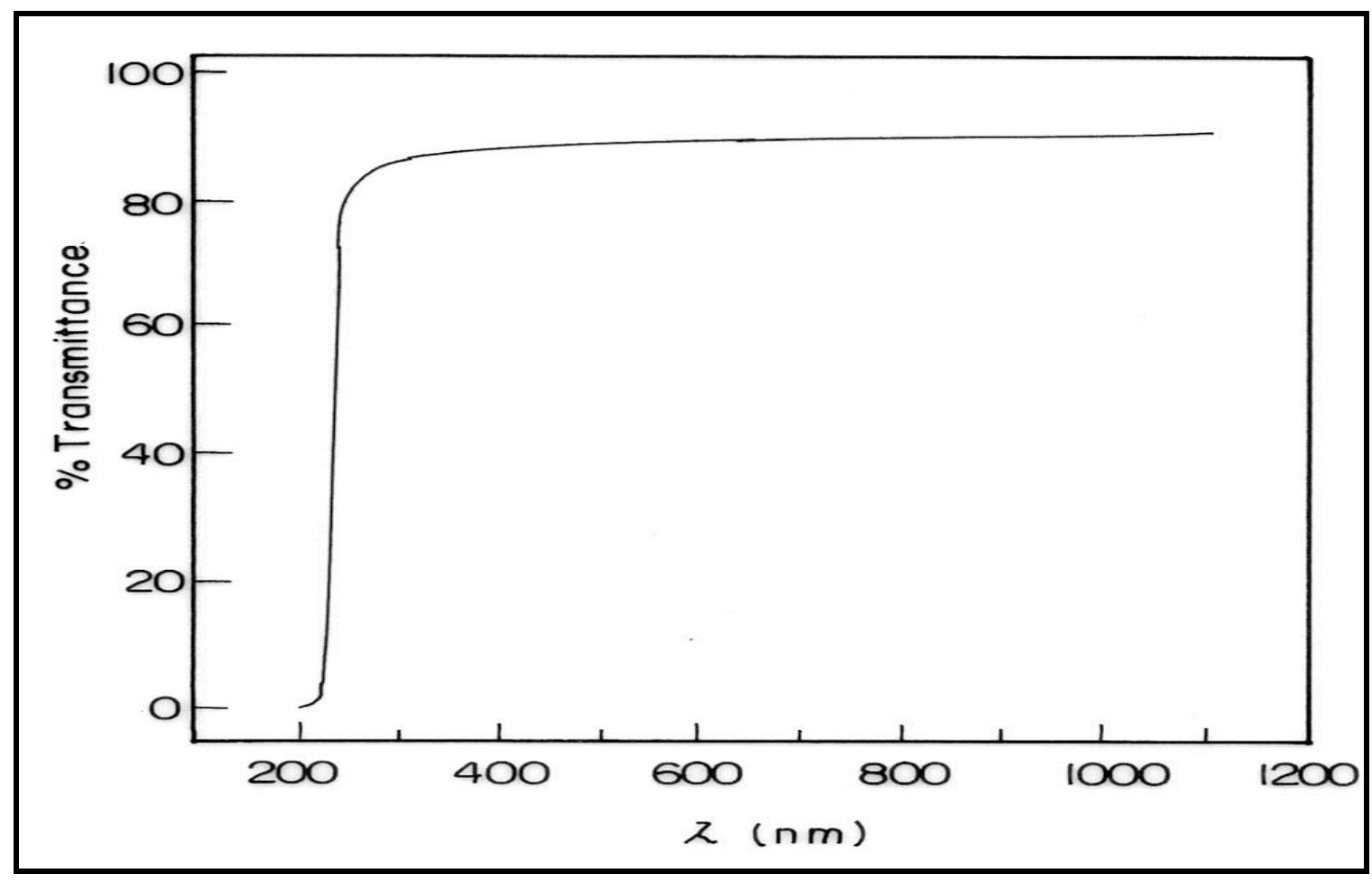

Figure 2 Transmission spectrum of L-Prolinium tartrate 


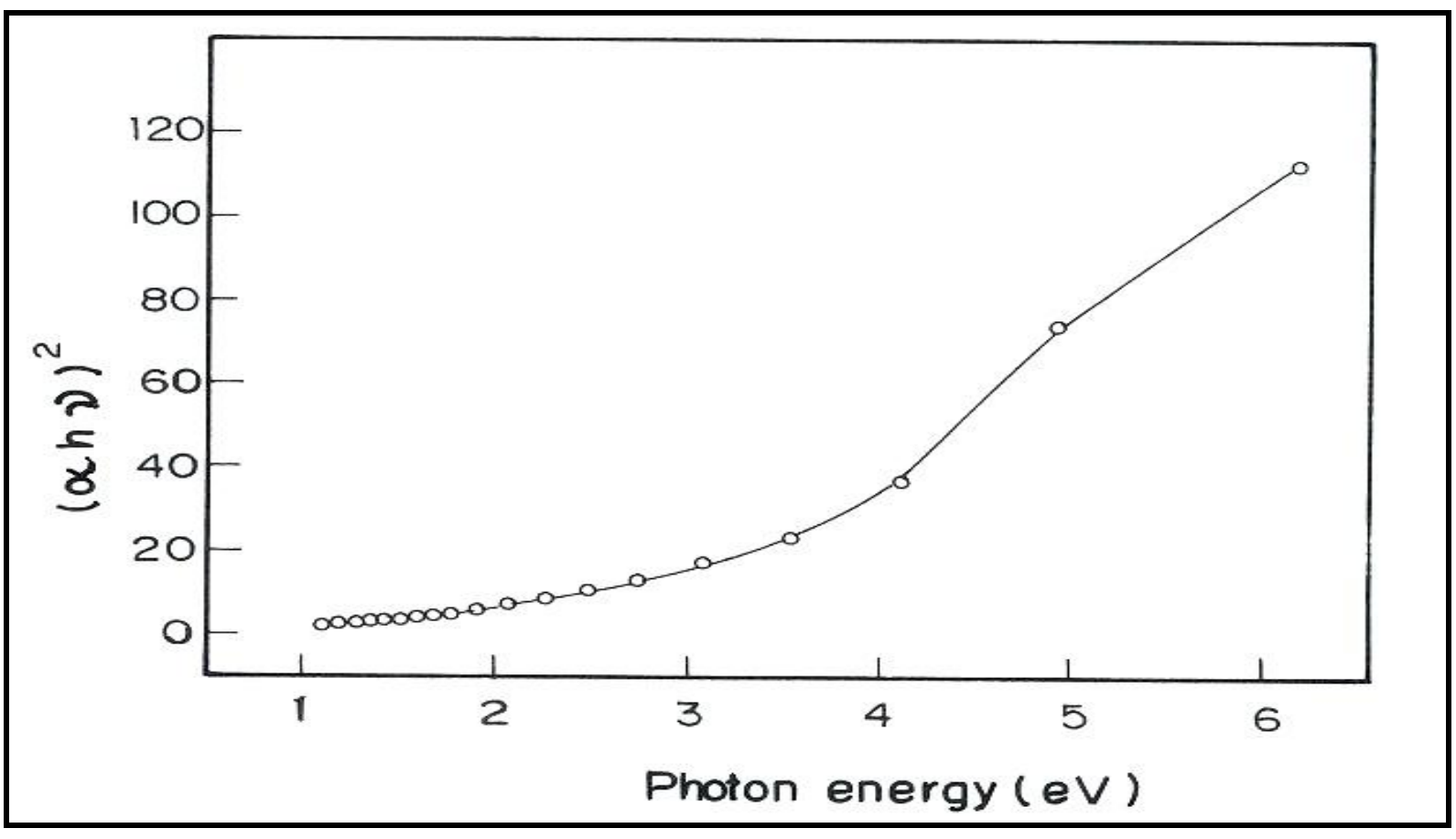

Figure 3 Plot of $(\alpha h v)^{2}$ versus hv for LPT single crystal

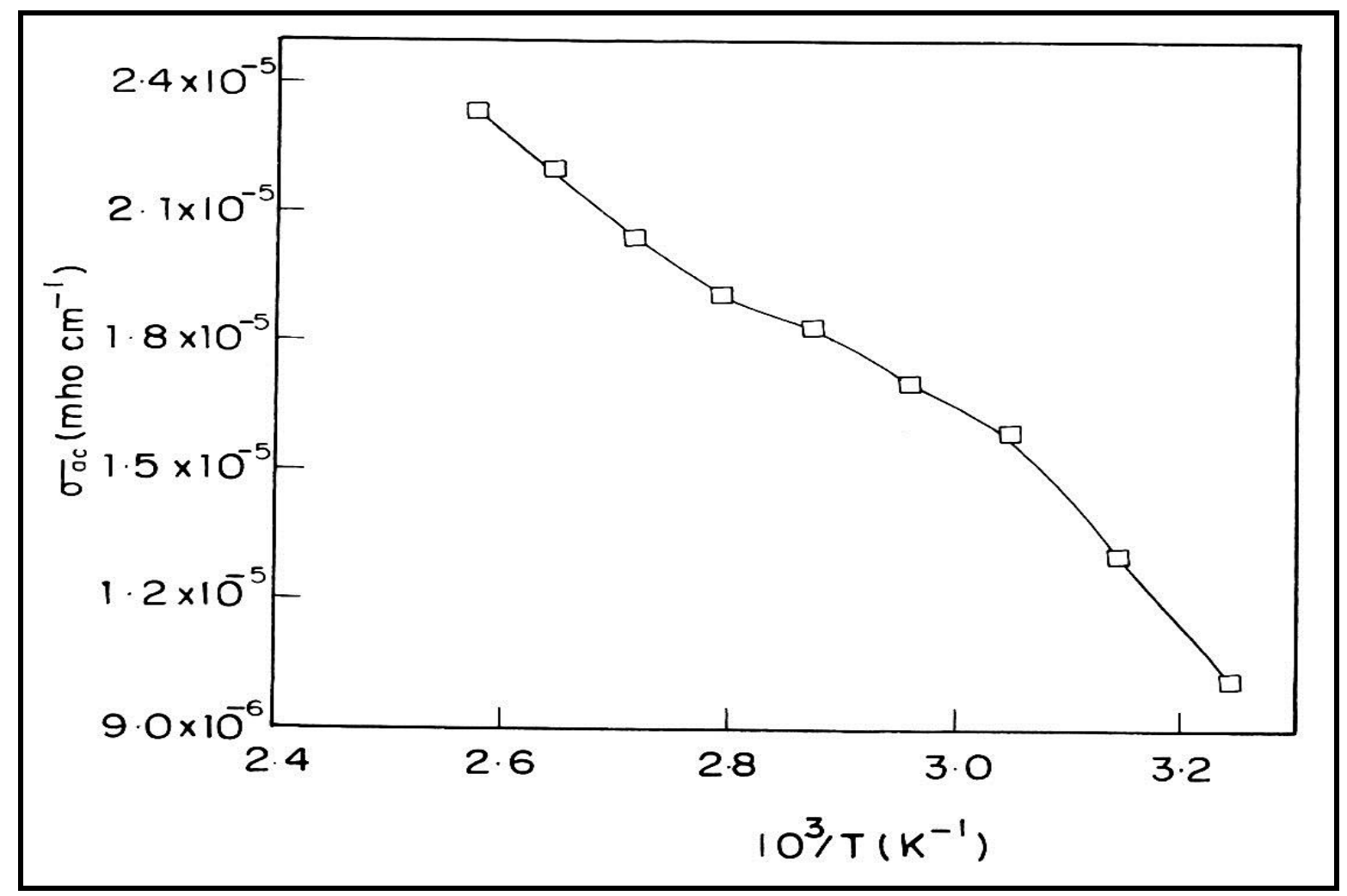

Figure.4 Variation of $\sigma_{\text {ac }}$ conductivity with 1000/T for LPT single crystal 


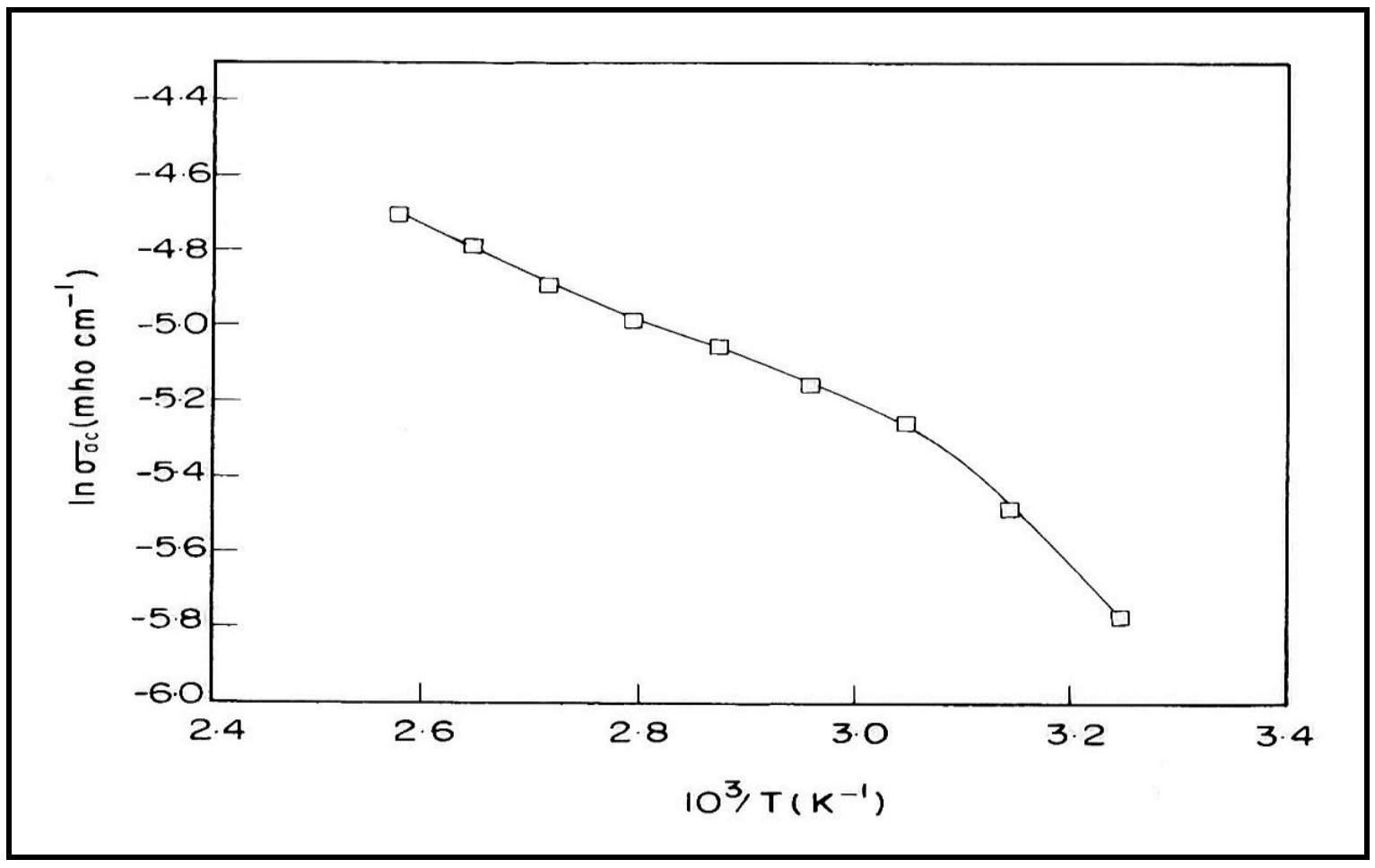

Figure. 5 Plot of $\ln \left(\sigma_{\mathrm{ac}}\right)$ versus 1000/T for LPT single crystal

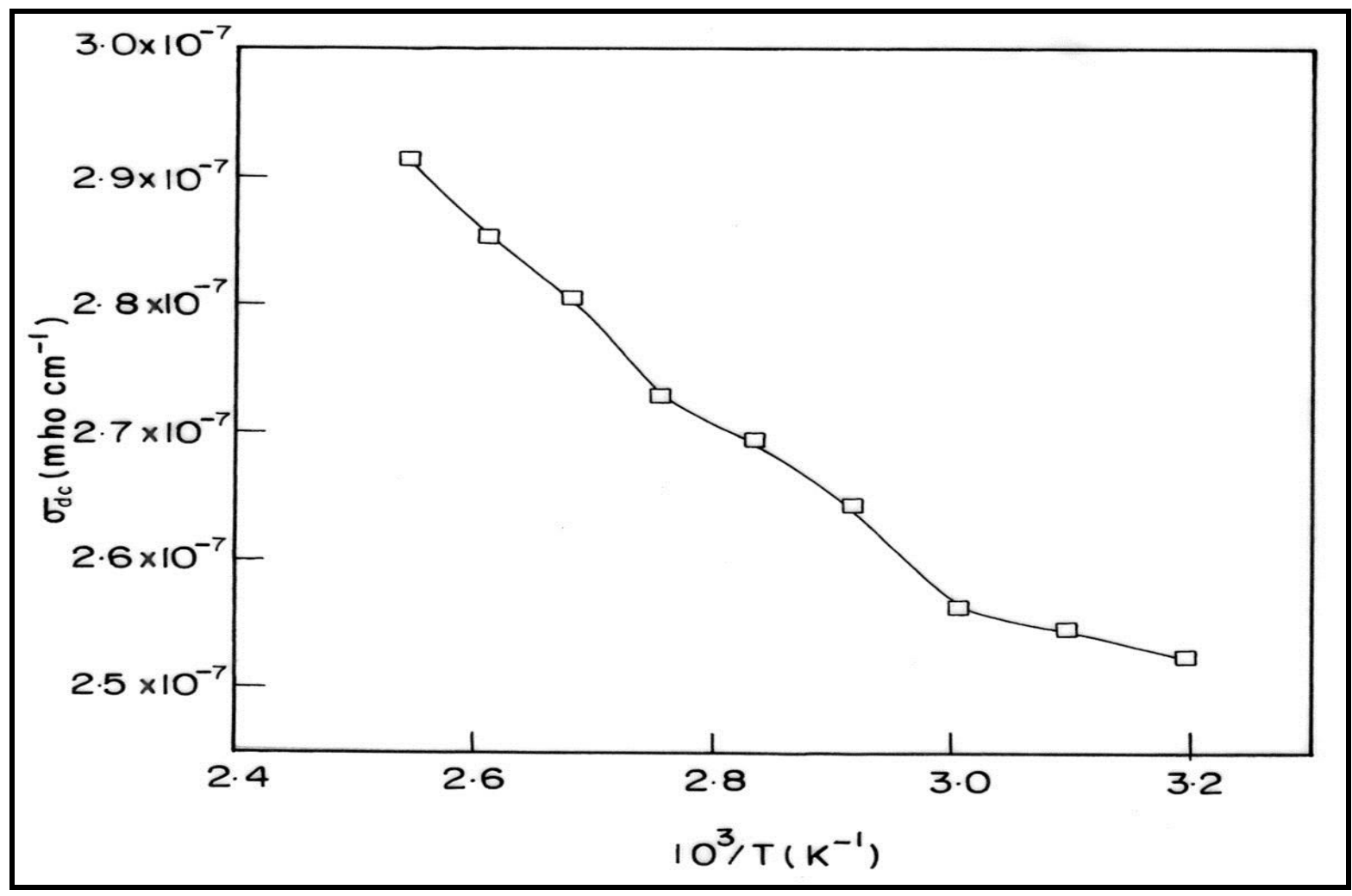

Figure 6 Variation of $\sigma_{\mathrm{dc}}$ conductivity with 1000/T for LPT single crystal 


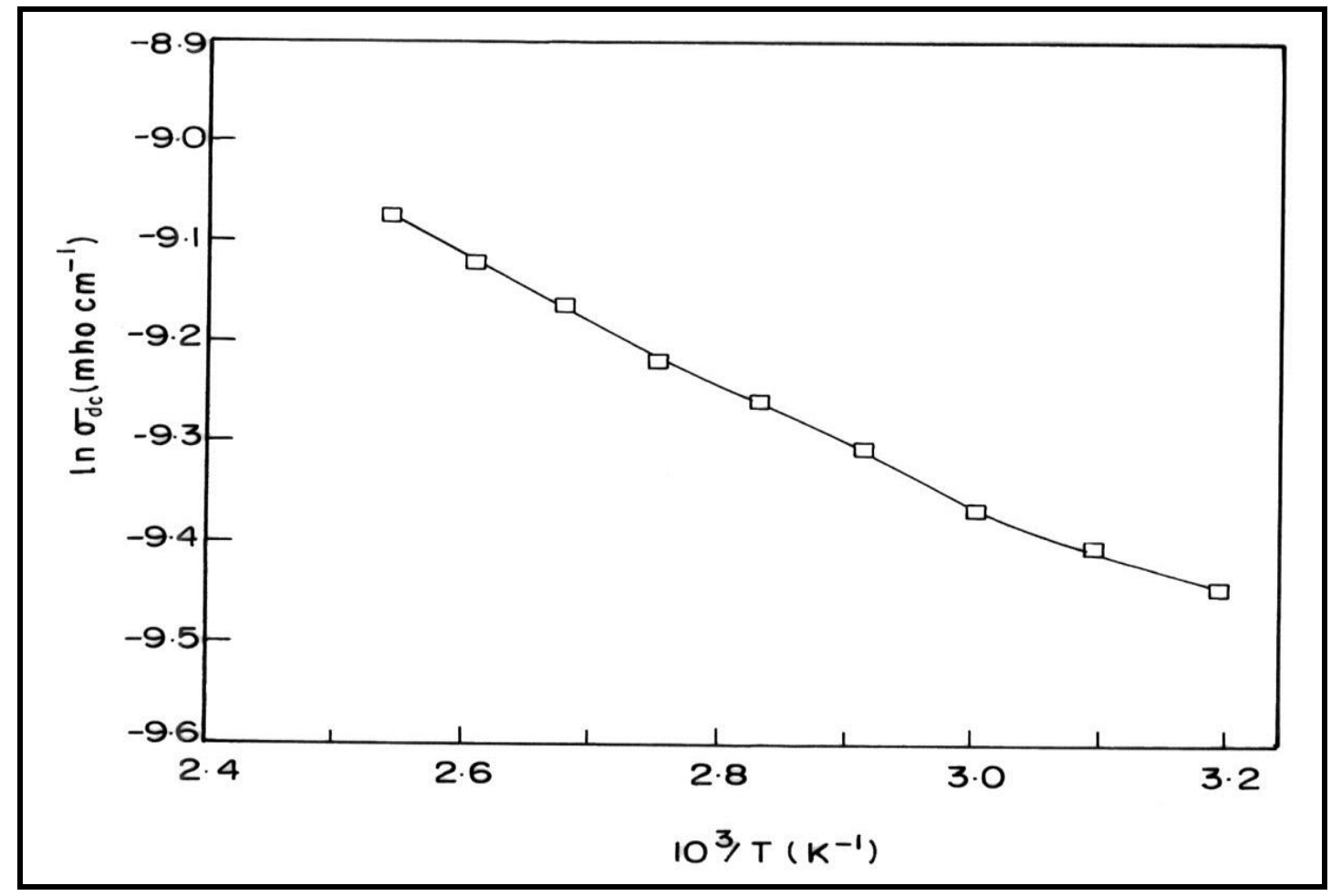

Figure .7 Plot of $\ln \left(\sigma_{\mathrm{dc}}\right)$ versus 1000/T for LPT single crystal

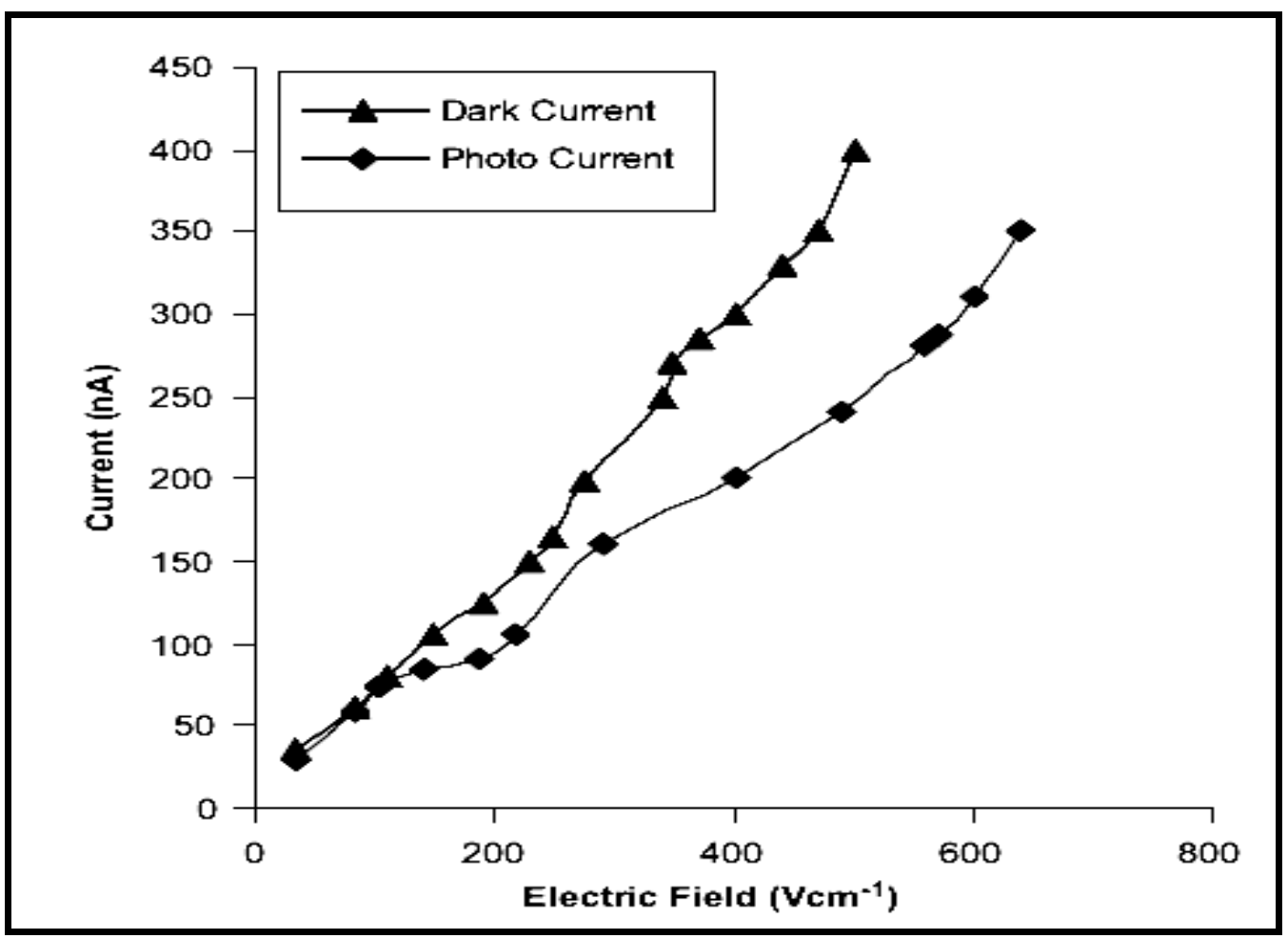

Figure 8 Field dependence of dark and photocurrents 


\section{CONCLUSION}

Single crystals of LPT were grown by using the slow evaporation technique. The lattice parameters were calculated by single crystal X-ray diffraction and it was confirmed that the crystals belong to the orthorhombic system with the space group $\mathrm{P} 2{ }_{1}$. Optical band gap $\left(E_{g}\right)$, absorption coefficient $(\alpha)$, extinction coefficient $(K)$, refractive index $(n)$, electric susceptibility $\chi_{c}$ and dielectric were calculated as a function of wavelength. The optical study is useful to determine the transparency region and the optical band gap and dielectric behavior of the material. The activation energy was determined from the plots of ac and dc conductivity. The photocurrent was less than the dark current, signifying negative photoconducting nature.

\section{REFERENCES}

[1] Y.Y. Lin, N.P. Rajesh, P. Santhanaragavan,
P. Ramasamy, Y.C. Huang,2002, Mater. Lett. 56, 1074.

[2] P.A. Franken, A. Hill, C.W. Peters, G. weinrich, 1961, Phys. Rev. Lett. 7, 118.

[3] Richard, P. Van Duyne, 2004, Science 306, 985.

[4] Martin Britto Dhas S.A, Natarajan S.,2007, Cryst. Res. Technol. Vol. 42, 471-476

[5] Ashour A, El-Kadry N, Mahmoud S A., 1995, Thin Solid Films. 269,117

[6] Gupta V, Mansingh A. 1996, J. Appl. Phys. 80,1063 .

[7] Gaffar MA, Abu El-Fadl A, Bin Anooz S, 2003. Physica B. 327,43 . 